\title{
CONTRÔLE DE LA PURETÉ DES SUBSTANCES ANESTHESIQUES!
}

\author{
J. G. Patorne, M.D., et J. C. Pouliot, M.D. ${ }^{2}$
}

La NÉCEsSité d'administrer les médicaments les plus purs possible est evidente. A plus forte raison, les substances anesthésiques doivent-elles être exemptes d'impuretés puisqu'elles s'adressent à des individus dont la résistance est plus ou moins ébranlée soit par la maladie en cause, soit par le choc, soit par l'agression chirurgicale. Les impuretés des substances anesthésiques, si elles ne compromettent pas toujours directement la vie du patient, réduisent pour le moins la valeur de l'anesthésie.

La plupart des anesthésistes ne disposent ni des moyens techniques ni de la formation rèquise pour contrôler personnellement la pureté des substances anesthésiques: Après avoir exposé notre opinion sur ce sujet, nous voulons rappeler à votre mémoire quelques tests de pureté facilement réalisables et surtout, signaler afin de les empêcher, les processus de contaminatiòn des substances anesthésiques.

Les impuretés peuvent y apparaitre en deux occasions: soit pendant la fabrication, soit pendant la conservation ou l'usage, par dégradation intrinsèque du produit ou par apport extérieur (1).

Il est assez rare que les substances anesthésiques en général soient le résultat de réactions chimiques simples. Leur fabrication nécessite souvent la présence de catalyseurs et le résultats en est fréquemment non pas un seul mais plusieurs produits qu'il faut séparer les uns des autres. D'autre part, il est pratiquement impossible pour les anesthésistes et les hôpitaux d'effectuer la vérification à ce stage. Il semble alors suffisamment prudent de se fier à la garantie d'un fabricant de bonne réputation, reconnu pour employer les meilleurs produits bruts; il entretient son propre laboratoire de vérification et observe les standards gouvernementaux requis:

La détérioration des substances anesthésiques pendant la conservation et pendant l'usage nous intéresse davantage parce que nous sommes souvent à même de la retarder sinon de l'empêcher: Elle survient par suite de l'action des agents physiques: air, lumière, température, humidité; ou par transformation chimique tel que oxydation, réduction hydrolyse, polymérisation, tautomérisation ou par combinaison d'un phénomène chimique et d'une activité physique (2).

Lors de son oxydation, un corps s'ajoute de l'oxygène ou perd de l'hydrogène; dans un cas comme dans l'autre, il y a perte d'électrons. La réduction est le phénomène inverse et la substance concernée s'enrichit d'électrons; ces deux phénomènes sont habituellement couplés. L'hydrolyse, c'est la décomposition d'une substance par ou en présence d'eau avec formation habituelle d'un acide et d'un alcool (3).

1Travail présenté à la réunion annuelle de la Société Canadienne d'Anesthésie le 5 mai, 1959.

2Du service d'anesthésie de l'Hópital de'l'Enfant-Jésus, Québec, P.Q. 
La combinaison entre elles de deux molécules ou plus d'un même corps chimique pour former une molécule plus lourde s'appelle polymérisation. Les aldéhydes possèdent cette tendance. Les cétones possèdent une autre propriété: la tautomérisatior: c'est le transfert d'un atome d'une position à une autre à l'intérieur d'une même molécule pour former un isomère (4).

Signalons aussi qu'un solide a moins tendance à se modifer qu'un liquide et ce dernier moins qu'un gaz (6).

Comme nous l'avons déjà dit, les normes de pureté des substances anesthésiques sont déterminées par de publications officielles et elles peuvent être recherchées par des moyens physiques ou chimiques (1). Cependant, il faut admettre que les tests simples d'identification sont à peu près inexistants. Il faut presque toujours combiner plusieurs tests d'élimination, ce qui permet d'identifier les substances par déduction.

Les principales propriétés physiques qui peuvent servir à établir le dégré de pureté sont: les points d'ébullition, de liquéfaction, de solidification, la recherche des résidus solides, la turbidité, la densité, l'indice de réfraction, la polarisation, la viscosité. Les moyens chimiques sont les pouvoirs de solubilité, de réduction, d'oxygenation, d'hydrolyse, dhalogénation et te $\mathrm{pH}$. Enfin, des tests biologiques pourraient être utilisés pour compléter l'information.

Certaines de, ces propriétés chimiques se manifestent par la formation d'un précipité ou par le changement de couleur d'un réactif. Ces phénomènes sont à la base des tests de puretés auxquels nous faisions allusion.

\section{Oxycìne}

La pureté de l'oxygìne employée en anesthésie doit être de 99 pour cent en volume d'oxygène. De plus, les 'ballons ne doivent contenir ni . Cb, ni $\mathrm{CO}_{2}, \mathrm{ni}$ halogènes. Etant donné que les normes de pureté exigées pour l $\mathrm{l} C \mathrm{o}^{\text {ne indus- }}$ triel sont supérieures à celles de l'oxygène médical, il n'y a pas de risque de confusion. Enfin, l'oxygène étant un gaz simple, il ne se décompose pas dans les conditions ordinaires de conservation.

\section{PROTOXYDE D'AZOTE}

\section{Préparation}

Le protoxyde d'axote se prépare habituellement en chauffant du nitrate d'ammonium à $240^{\circ} \mathrm{C}$. (5). C'est le premier d'une séries d'oxydes d'azote où cet élément a une valence de plus en plus élevée. La toxicité augmente proportionnellement à la valence de l'azote en combinaison avec l'oxygène. Or, ces oxydes peuvent se furmer en cours de fabrication. Lorsqu'ils sont inhales, ils se combinent avec l'eau tissulaire pour former des acides toxiques pour l'épithélium alvéolaire. La manifestation clinique en est un œdème pulmonaire.

D'autre part, l'oxyde nitrique (NO) peut se combiner avec l'hémoglobine d'une manière préférentielle, à la façon du monoxyde de carbone (CO) pour ainsi empêcher le transport de l'oxygène. 
Si le protoxyde d'azote contient de leau, il en résulte deux inconvénients: (i) l'eau se condense sur les valves des appareils lors de la détente, qr modifie le débit gazeux (7); (ii) l'eau contribuel à la formation d'acides en présence de peroxydes.

\section{Dépistage}

Nous disposons de deux tests:

Test au sulfate ferreux ( $\left.\mathrm{Fe} e \mathrm{SO}_{4}\right)$. Il s'agit de faire passer un échantillon du gaz dans une solution de sulfate ferreux. Le présence d'oxyde nitrique se manifeste

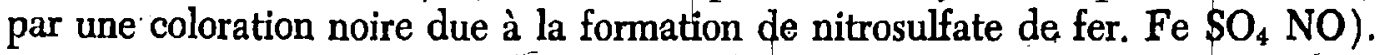
Cependant, cette réaction est réversible et la coloration peut disparaître après un certain temps (Transparent 3-4).

Test d liodure de potassium. On peit déceler les oxydes supérieurs au protoxyde d'azote en faisant barbotter un specimen dans une solution d'amidon et d'iodure de potassium $15 \mathrm{M}$ contenant une goutte d'acide acétique glacial. Les peroxydes oxydent l'iodure de potassium et l'iode qui s'en libère produit une coloration bleue en présence de la solution d'empois d'amidon. Ce test présente l'avantage de déceler tous les peroxydes, tandis que le précédent se limite à l'oxyde nitrique.

Retenons enfin, que le protoxyde d'azote ne se polymérise pas et ne se transforme pas spontanément en d'autres produits. La pharmacopée américaine exige qu'il soit pur à 95 pour cent.

\section{CyCLOPROPANE (TRIMÉTHYL.̇̀ne)}

\section{Préparation}

Là préparation du cyclopropane exige plusieurs étapes. Disons simplement que la dernière de ces étapes consiste à faire chauffer du 1 chloro-3 bromo propane en présence de zinc. D'après les exigences de la pharmacopée américaine, sa pureté doit être de 99 pour cent, en volume.

\section{Impuretés}

Le cyclopropane se comporte à la fois comme un hydrocarbone saturé et comme un non saturé. Il résiste bien à l'oxydation et les alcalins ont peu d'effet sur lui. Cependant, il se transforme assez facilement en son isomère, le propylène, surtout en présence d'un catalyseur comme la limaille de fer et à température élevée. On peut aussi trouver dans le cyclopropane, du cyclohexane, de l'azote, du $\mathrm{CO}$ du $\mathrm{CO}_{2}$ et des-halogènes complexes: v.g., chlorpropane. Mais le propylène demeure l'impureté la plus susceptible d'être rencontrée car il peut se former aussi bien pendant la fabrication que pendant la conservation.

\section{Dépistage}

Propylène. On recherche le propylène en faisant barbotter -un échantillon dú gaz dans une solution alcaline de permanganate de potassium. L'oxydation du propylène aux dèpens du permanganate de potassium fait passer ce dernier de pourpre qu'il était au brun (8). Cette réaction est aussi indicatrice d'autres oléfines. 
Bioxyde de carbone (Transparent no 2). Au lieu de rechercher le $\mathrm{CO}_{2}$ par la méthode classique l'hydroxyde de baryum, nous avons utilisé une solution aqueuse de chaux sodée avec indicateur. Nous y avons fait barbotter le cyclopropane suspect. Comme aucune coloration n'est apparue, nous avons conclus qu'il était exempt de $\mathrm{CO}_{2}$. En contre preuve, on a fait expirer dans une solution identique

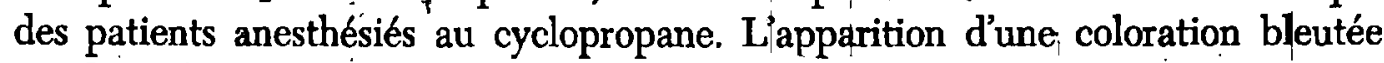
a indiqué la présencé de $\mathrm{CO}_{2}$ dans les gaz d’expiration.

Monoxyde de carbone. Il faut d'abord préparer une solution d'hémoglobine en ajoutant $2.5 \mathrm{ccc}$. de sang à $50 \mathrm{cc}$. d'une solution contenant $25 \mathrm{mg}$. d'ac. tannique et $25 \mathrm{mg}$. d'ac. pyrogallique. On y expose ensuite $250 \mathrm{cc}$. du gaz à analyser. L'oxyhémoglobine et l'hémoglobine réduite vont réagir réciproquement avec l'ac. tannique et l'ac. pyrogallique pour former un précipité brun. Si l'échantillon contient du monoxyde de carbone, la carboxyhémoglobine qui en résulte étant plus stable ne réagit pas avec ces acides, et retient sa coloration rosée.

\section{ETHER-ÉTHYLIQQUE}

\section{Fabrication}

La façon courante de fabriquer l'éther anesthésique consiste à déshydrater par chauffage l'alcool éthylique en présence d'acide, sulfurique concentré.

\section{Impuretés}

Nous divisions en deux classes les impuretés que nous pouvons retrouvier dans l'éther. D'abord, les impuretés de fabrication, notamment l'acide sulfurique, l'alcool éthylique et leurs dérivés. On comprend en effet que des molécules des ingrédients de base puissent passer dans le produit firal. Dans le second groupe, nous reconnaissons les impuretés de conservation. Ce sont les peroxydes d'éther, les aldéhydes et plus spécifiquement l'acétaldéhyde. La formation de ces produits de décomposition sera favơrisée par l'air, la lumière et la chaleur, tandis qu'elle sera retardée par les métaux lourds, en particulier le cuivre, et aussi par la diphenylamine et l'hydroquinone (9).

On considère comme satisfaisant un taux d'alcool ne dépassant pas 4 pour cent. D'ailleurs, l'alcool présente l'avantage d'élever le point d'ébullition de l'éther, avantage dont on tire profit dans les climats chauds et les hautês altitudes.

L'acide sulfurique dans l'éther déclenche, ou tout au moins favorise en présence d'alcool, la formation de peroxydes qui par la suite cèdent leur place aux aldéhydes. Certains auteurs prétendent que l'éther contenant des ces impuretés est toxique pour les; cellules respiratoires ciliées. De toute façon, il est certain que sa puissance anesthésique est diminuée par leur présence. Par contre, d'autres auteurs, ont troưvé que 0.5 pour cent d'aldéhyde est sans effet chez les animaux d'expérience et qu'une concentration égale de peroxydes produirait tout au plus une légère irritation gastrique chez l'homme.

\section{Dépistage}

$p H$. L'éther doit avoir une réaction neutre. Si on mélange une égale quantité d'eau distillée et d'éther, les deux phases doivent rester neutres. On devra sus- 
pecter la présence d'acide sulfurique si le papier tournesol bleu vire au rouge: Peroxydes (Transparent no 1). Le dépistage des peroxydes se fait de la façon suivante: ajouter $1 \mathrm{cc}$. d'une solution à 10 pour cent d'iodure de potassium à $10 \mathrm{cc}$. d'éther. Laisser reposer à la noirceur dans un contenant hermétique durant trente minutes. La présence de peroxyde se manifeste par une coloration jaune due à la libération de l'iode élément. Si l'on veut contrôler le résultat dẹ cette réaction on peut y ajouter quelques goúttes d'une solution d'empóis d'amidon. Cette solution en présence d'iode libre prendra une coloration bleu foncé (10).

Aldéhydes. Pour la recherche des aldéhydes on met à profit leur capacité de réduire le mercure de la solution de Nessler. Après avoir agité $20 \mathrm{cc}$. d'éther et $3 \mathrm{cc}$. du réactif, on laisse former deux couches. Une coloration jaune de la partie aqueuse (i.e., solution de Nessler) indique la présence d'aldéhydes qui, en quantité suffisante, pourront former un précipité jaune.

Il faut se méfier d'une réaction faussement positive. A la longue, l'alcool contenu dans l'éther s'oxyde aux dépens du mercure. Ce dernier étant réduit pourra se colorer ou encore précipiter.

\section{ChLoroforme}

\section{Préparation}

On prépare le chloroforme par réduction du tétrachlorure de carbone en présence de fer comme catalyseur. On y ajoute 1 pour cent d'alcool éthylique pour fins de stabilisation.

\section{Impuretés}

Parmi les impuretés que l'on peut retrouver dans le chloroforme, signalons l'acide chlorydrique, certains aldéhydes, de l'acétọe, et enfin un dérive cétonique très dangereux, le phosgène.

\section{Dépistage (Transparent no 5)}

L'eau distillée, après lavage de chloroforme exempt des impuretés ci-haut nommées, restera neutre au papier tournesol. Les corps cétoniques, le phosgène et les aldéhydes produiront une réaction positive en présence de la solution de Nẹsler.

Si l'on soupçonne la présence d'halogènes, on pourra les faire précipiter par une solution acidifiée de nitrate d'argent.

\section{TRICHLORÉTHYLÈNE}

Les produits de décomposition du'trichloréthylène sont 'les mêmes que ceux du chloroforme, et nous pouvons recourir aux mêmes tests de dépistage.

\section{Discussion}

Dans ce court exposé, nous navons considéré que les anesthésiques les plus usuels. Depuis plusieurs mois, nous avons pratiqué à maintes reprises les tests 
ci-haut décrits. Nous n'avons pas réussi à trouver d'impuretés dans les différents échantillons.

C'est dans le but d'illustrer les différents tests que nous avons contamibé volontairement certains de nos échantillons. Même si à date nous n'avons pas décelé d'impuretés, nous nous proposons de continuer à pratiquer ces teșts $\mid$ a loccasion, ne serait-ce que pour garder en mémoire les principes suivants:

TABLEAU I

\begin{tabular}{|c|c|c|c|c|c|c|c|c|c|c|}
\hline \multirow[b]{2}{*}{ Agents } & \multicolumn{10}{|c|}{ Impuretes } \\
\hline & Peroxydes & s Aldéhıydes & Acêtylène & $\begin{array}{l}\text { Hydro- } \\
\text { carbures } \\
\text { halogénés }\end{array}$ & $\mathrm{CO}$ & $\mathrm{co}$ & $\begin{array}{l}\text { Olefiney } \\
\text { (propy- } \\
\text { lene) }\end{array}$ & Phosgène & $\mathrm{HCl}$ & $\begin{array}{c}\text { NO } \\
\text { (peroxydes } \\
\text { d'axote) }\end{array}$ \\
\hline \multicolumn{11}{|l|}{ Diethyléther } \\
\hline$\left(\mathrm{C}_{2} \mathrm{H}_{\mathrm{b}}\right) \mathrm{O}\left(\mathrm{C}_{2} \mathrm{H}_{\mathrm{s}}\right)$ & $\mathbf{X}$ & . $\mathbf{x}$ & & & & & & & & \\
\hline Divinyléther & & - & & & & & & & & \\
\hline$\left(\mathrm{C}_{2} \mathrm{H}_{3}\right) \mathrm{O}\left(\mathrm{C}_{2} \mathrm{H}_{3}\right)$ & $\mathbf{x}$ & $\mathrm{x}$ & $x$ & & & & & & & \\
\hline Protoxyde & & & & & & & & & & \\
\hline d'azoie $\mathrm{N}, \mathrm{O}$ & & & & & & & & & & $\mathbf{x}$ \\
\hline \multicolumn{11}{|l|}{ Triléne } \\
\hline $\mathrm{C}_{2} \mathrm{HCl}$ & & $\mathrm{x}$ & & & & & & $\mathbf{x}$ & $\mathbf{x}$ & \\
\hline Chloroforme & & & & & & & & $\therefore$ & & \\
\hline $\mathrm{CHCl}_{\mathbf{3}}$ & & $x$ & & $x$ & & & & $\mathbf{x}$ & & \\
\hline Cyclupropane & & & & & & & & & & \\
\hline $\mathrm{C}_{3} \mathrm{H}_{6}$ & & & & $\mathbf{x}$ & $\mathbf{x}$ & $\mathbf{x}$ & $\mathrm{x}$ & & & \\
\hline
\end{tabular}

1. L'éther, le ehloroforme et le trilène sont les substances dont la conservation exige le plus d'attention car leur mode d'administration nécessite l'exposition aux agents physiques. C'est ainsl que l'on doit considérer comme suspect l'éther qui a séjourné dans une boite ouverte depuis une semaine ou dans un vaporisateur depuis deux jours.

2. Le cyclopropane et le protoxyde d'azote sont relativement stables 'pendant leur conservation à condition d'avoir été livrés purs.

3. Les produits de décomposition les plus dangereux sont: le phosgène, l'oxyde nitrique (et perroxydes d'azote), les aldéhydes.

4. En plus de leur toxicité, les peroxydes d'éther comportent certains dangers d'explosion: les vaporisateurs devraient être nettoyés fréquemment car des peroxydes peuvent y demeurer après évaporation de l'anesthésique.

5. L'ôdeur inusitée d'une substance doit faire craindre la présence d'impuretés.

6. Il ne faut pas hésiter à rejeter une substance douteuse.

\section{Conciusion}

Nous avons voulu faire une brève revue des principales impuretés qu'on peut rencontrer dans les substances anesthésiques les plus employées.

Il est étonnant de constater qu'avec six ou sept réactiọns, on peut décelef la majorité des impuretés. susceptibles de se former. 'S'ils ne sont pas hautement spécifiques, ces tests ont l'avantage d'être fort simples tout en ayant une valeur suffisante pour commander l'abandon d'une substance à réaction positive.| Ils permettent ainsi d'aughnenter la marge de sécurité de l'anesthésie moderne. 
TABLEAL II.

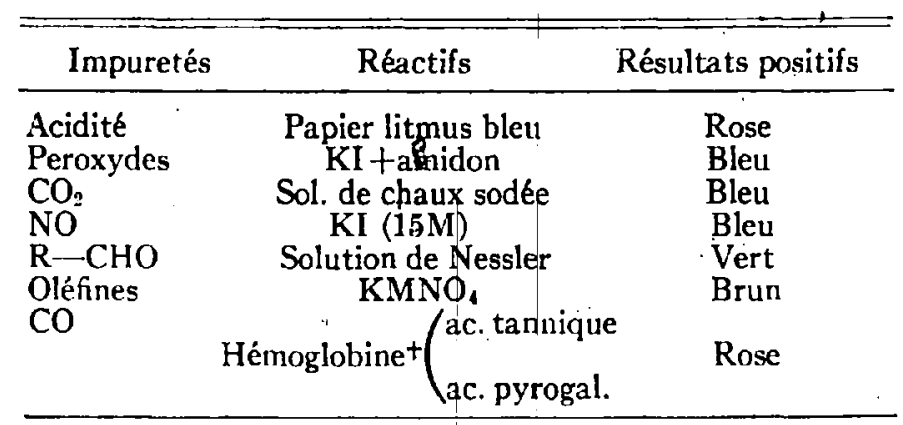

\section{SUMmaRY}

During the last few months we have had tne opportunity to check the purity of the following anaesthetics: nitrous oxide, cyclopropane, ether, chloroform, trichloroethylene. We have taken advantage of this to review the principal tests used in this regard. Up to now, we have not found any impurities in the samples examined. However, we have on purpose contaminated some of these in worder to prove without doubt the results. of the principal reactions.

Depending on the individual anaesthetic, peroxides, propylene, carbon monoxyde, carbon dioxide, aldehydes, and phosgene are the impurities most likely to be found. It is possible with a few simple tests to detect most impurities. We believe that the frequent practice of these tests would widen the security margin of modern anaesthesiology.

\section{BIBLIOGRAPHIE}

1. Adrun, J. The Chemistry of Anesthesia. Springfield; Ill.: Charles C. Thomas (1954).

2. Anhian, J. The Pharmacology of Anesthetic Drugs, Springfield, Ill.: Charles C. Thomas (1856).

3. Wertemen, E. Introductory Organic Chemistry. Philadelphia: Blakiston (1946).

4. Gagné. Traité élémentaire de de chimie. (1955).

5. Lee, AlFred. Synopsis of Anaesthesia: Wright (1957).

6. Becrman. Drugs: Their Nature, Action and Use. Philadelphia: W. B. Saunders (1958).

7. Macnstosn \& Musims. Physics for the Anesthetist. Springfield, Ill.: Charles C. Thomas (1958).

8. Bunger, O. K. Tests for Impurities in Cyclopropane. Anesth. \& Analg. 4: 207 (July-Aug. 1937).

" 9. Gol., H., \& Gold, D. The Stability of U.S.P. Ether after the Metal Container has been Opened: With Preliminaryy Results of a Clinical Cpmparison of U.S.P. Ether in Large Drums with Ether in Small Cans Labeled "'For Anesthesia." Anesth. \& Analg. 2: 92 (March-April 1935).

10. Schurys, J. G., \& NeELEY, A. H. Studies on the Formation and Decomposition of Ether Peroxides. Anesthesiology 19 (5): (Sept.-Oct. 1958). 